\title{
Análise de vibrações em vigas com buracos prescritos
}

\author{
Lucas dos Santos Fernandez ${ }^{1}$ \\ Laboratório Nacional de Computação Científica, LNCC/MCTIC, Petrópolis, RJ, Brasil \\ Tatiane Weimann ${ }^{2}$ \\ Programa de Pós-Graduação em Modelagem Matemática, IFM/UFPel, Pelotas, RS, Brasil \\ Anderson Martins ${ }^{3}$ \\ Programa de Pós-Graduação em Modelagem Matemática, IFM/UFPel, Pelotas, RS, Brasil \\ Alexandre Molter ${ }^{4}$ \\ Departamento de Matemática e Estatística, IFM/UFPel, Pelotas, RS, Brasil
}

\begin{abstract}
Resumo. O aparecimento de vibrações em estruturas, mediante movimento, sempre merece a atenção dos projetistas. Vibrações podem ser indesejadas quando afetam a vida útil da estrutura ou danificam o seu entorno. Neste caso, há necessidade de controle das vibrações, o que pode ser feito por meio de um controle ativo, passivo, ou de ambos simultaneamente. Por outro lado, vibrações podem causar grandes deformações estruturais, que por sua vez podem ser úteis na conversão e produção de energia. De qualquer forma, é necessário o conhecimento da dinâmica estrutural. Portanto, o objetivo deste trabalho é analisar vibrações em estruturas do tipo vigas em balanço, onde são inseridos buracos em formato elíptico, em diferentes quantidades. O Método de Elementos Finitos (MEF) é utilizado para a discretização do domínio da estrutura e as vibrações são analisadas por meio de análise modal. São feitas comparações da amplitude de vibração para o primeiro modo, considerando um, dois e três buracos na viga. Os resultados desta análise são apresentados e discutidos.
\end{abstract}

Palavras-chave. Viga com Buracos, Dinâmica, Modos de Vibração, Amplitude.

\section{Introdução}

O conhecimento e a análise de vibrações que aparecem na dinâmica de estruturas é muito importante, uma vez que vibrações indesejadas e de grande amplitude podem danificar as estruturas ou o seu entorno, como por exemplo, bancadas de apoio, engates ou paredes. Depois que se toma conhecimento das vibrações, é possível a utilização de meios para o controle das mesmas, ou ainda, fazer uso delas para geração de energia.

O controle de vibrações pode se dar por meio de controle ativo, através da utilização de atuadores, ou passivo, sem o uso dos mesmos. Ao se considerar estruturas do tipo vigas, uma forma de controle passivo que vem emergindo é a inserção de buracos na estrutura $[2,6,8]$. No que concerne à conversão de energia, utiliza-se coletores, normalmente materiais piezelétricos, que são fixados ou embutidos em locais onde há maior deformação estrutural. Uma maior deformação pode ocorrer nas extremidades dos buracos que são inseridos nas estruturas. Para conversão de energia (mecânica em elétrica), é fundamental ter-se conhecimentos da dinâmica estrutural, o que inclui a análise de vibrações $[1,3]$, para então podermos projetar mecanismos de coleta da energia gerada pela estrutura por meio de suas vibrações.

\footnotetext{
${ }^{1}$ lucasf@lncc.br

2 tatiane.weimann@gmail.com

3 andersondmm@hotmail.com

4 alexandre.molter@ufpel.edu.br
} 
Tendo em vista a necessidade do conhecimento da dinâmica estrutural, tanto para o caso do controle passivo como para a conversão de energia (a conversão ocorre nos coletores de energia), ambos com propósitos diferentes, percebe-se que uma análise de vibrações em estruturas para diferentes formatos e quantidade de buracos se torna indispensável. Sendo assim, o propósito deste trabalho é analisar as vibrações de uma viga em balanço, considerando 1, 2 e 3 buracos centralizados e computando o primeiro modo de vibração. Será feita uma comparação das amplitudes de vibração, considerando-se o primeiro modo de vibração para a viga sem buraco e com 1, 2 e 3 buracos. Naturalmente, a frequência natural será diferente para cada formato estrutural (sem e com buracos), uma vez que a massa estrutural é alterada. No entanto, o foco será a amplitude de vibração, pois menores amplitudes são um indicativo do uso desta configuração para um posterior controle de vibrações. Além disto, as bordas mais finas dos buracos, diante do movimento vibratório, contém deformações estruturais maiores do que o restante da estrutura, sendo interessante para a colocação de coletores de energia.

O texto está organizado como segue. A modelagem da dinâmica da viga é apresentada na Seção 2. Esta seção é constituída de duas partes: na primeira delas, o domínio da estrutura é discretizado em elementos finitos e faz-se uso do MEF para a obtenção da dinâmica; enquanto que na segunda parte é utilizada a análise modal para determinação das frequências e dos modos de vibração. Ainda serão utilizadas as equações em forma de espaço de estados e as amplitudes de vibração obtidas pela Transformada de Fourier. Na Seção 3 são apresentadas as simulações computacionais para ilustrar os resultados que são então discutidos. O trabalho termina com as conclusões e alguns comentários finais na Seção 4.

\section{Modelagem da dinâmica da viga}

Para a análise da dinâmica da viga é utilizado o MEF na discretização do domínio e cálculo dos deslocamentos nodais, e a análise modal para a obtenção dos modos de vibração.

\subsection{Discretização do domínio e cálculo dos deslocamentos nodais}

Considerando o estado de deformação plana, o domínio da estrutura utilizado neste trabalho foi discretizado usando o MEF, e a estrutura considerada foi uma viga engastada em um lado. A ideia é analisar a equação de equilíbrio de cada elemento, encontrar a equação local e, em seguida, correlacionar a contribuição de cada elemento à equação global. De acordo com [4], para a análise dinâmica do elemento, a equação governante do elemento (local) pode ser descrita como

$$
M_{e} \ddot{u}_{e}+C_{e} \dot{u}_{e}+K_{e} u_{e}=f_{e},
$$

sendo $u_{e}$ o vetor de deslocamento nodal do elemento, $f_{e}$ o vetor de força, e as matrizes $M_{e}, K_{e}$ e $C_{e}$ a massa, a rigidez e o amortecimento da estrutura, respectivamente. Tais vetores e matrizes são definidos como

$$
\begin{aligned}
f_{e} & =\int_{\Omega_{e}} N^{\top} b_{e} d \Omega_{e}+\int_{\Gamma_{e}} N^{\top} t_{e} d \Gamma_{e} \\
M_{e} & =\int_{\Omega_{e}} \rho N^{\top} N d \Omega_{e} \\
K_{e} & =\int_{\Omega_{e}} B^{\top} c B d \Omega_{e} \\
C_{e} & =\alpha K_{e}+\beta M_{e}
\end{aligned}
$$


onde $N$ representa as funções interpoladoras, $b_{e}$ e $t_{e}$ são vetores que retratam as forças de corpo e as forças superficiais atuando na estrutura, respectivamente, $B$ é a matriz de relação entre a tensão e o deslocamento e $c$ é a matriz constitutiva do material. As contantes $\alpha$ e $\beta$ são determinadas de acordo com o modelo de Rayleigh e a constante $\rho$ é a densidade do material.

A equação global que governa o domínio inteiro da estrutura é semelhante à Equação (1), tendo que levar em conta as apropriadas conexões dos elementos. Como neste trabalho foi considerado a análise das vibrações livres, os vetores de forças de corpo e de forças superficiais são nulos. No entanto, de forma geral, considerando $f$ o vetor de força, a equação global dinâmica da estrutura apresentada pode ser descrita na forma

$$
M \ddot{u}+C \dot{u}+K u=f .
$$

As matrizes globais $M, C, K$, continuam representando a massa, amortecimento e rigidez, respectivamente, e são encontradas associando-se a contribuição de cada elemento da viga, bem como o vetor $u$, dos deslocamentos nodais (na direção $x$ e na direção $y$ ) de toda a estrutura.

\subsection{Análise modal}

Com o intuito de facilitar a resolução da Equação (6), usou-se a superposição modal, pois ao utilizar as coordenadas modais $\eta$, é possível desacoplar as equações regentes do movimento, sendo que, conforme Gawronski [7], a representação por coordenadas modais pode ser obtida pela transformação do modelo nodal. Essa transformação é feita empregando a matriz modal que é determinada a partir da solução modal, sendo ela, o resultado do problema de autovalores e autovetores descrito pela equação matricial

$$
\left(K-\omega^{2} M\right) \Phi=0 .
$$

Os autovalores encontrados $\omega^{2}$, são denominados frequências naturais do sistema e são as frequências com que as vibrações livres ocorrem na estrutura. Já os autovetores, obtidos a partir da matriz $\Phi$ e associados aos autovalores encontrados, são nomeados modos de vibração e descrevem o formato e a amplitude associados às frequências naturais. A matriz modal $\Phi$ é a matriz com todos os modos do sistema, entretanto, como geralmente apenas os primeiros modos contêm informações relevantes à análise do sistema, utiliza-se a matriz modal truncada com $n$ modos.

Utilizando a matriz modal truncada, é possível transformar o vetor de deslocamento nodal em função das coordenadas modais, através da relação

$$
u=\Phi \eta \text {. }
$$

Substituindo a Equação (8) na Equação (6), obtém-se que

$$
M \Phi \ddot{\eta}+C \Phi \dot{\eta}+K \Phi \eta=f .
$$

Multiplicando todos os termos da Equação (9) por $\Phi^{\top}$, tem-se que

$$
\underline{M} \ddot{\eta}+\underline{C} \dot{\eta}+\underline{K} \eta=\underline{f},
$$

onde

$$
\underline{M}=\Phi^{\top} M \Phi, \quad \underline{C}=\Phi^{\top} C \Phi, \quad \underline{K}=\Phi^{\top} K \Phi \quad \text { e } \quad \underline{f}=\Phi^{\top} f .
$$

Multiplicando todos os termos da Equação (10) por $\underline{M}^{-1}$, obtém-se a equação

$$
\ddot{\eta}+Z \dot{\eta}+\Omega \eta=\underline{M}^{-1} \underline{f}
$$


na qual

$$
Z=\underline{M}^{-1} \underline{C}=\operatorname{diag}\left(2 \zeta_{i} \omega_{i}\right) \quad \text { e } \quad \Omega=\underline{M}^{-1} \underline{K}=\operatorname{diag}\left(\omega_{i}^{2}\right),
$$

onde o índice $i$ representa o número de modos de vibração a serem considerados.

Considerando como variáveis de estado o deslocamento e a velocidade modal, $\xi=\left[\begin{array}{ll}\eta & \dot{\eta}\end{array}\right]^{\top}$, pode-se reescrever a Equação (12) na representação em espaço de estados, a saber, na forma

$$
\dot{\xi}=A \xi+B f
$$

onde

$$
A=\left[\begin{array}{cc}
0_{n \times n} & I_{n \times n} \\
-\Omega & -Z
\end{array}\right] \quad \text { e } \quad B=\left[\begin{array}{l}
0_{n \times 1} \\
\underline{M}^{-1}
\end{array}\right]
$$

no qual $A$ é a matriz do sistema e $B$ é a matriz de controle.

\section{Resultados e discussões}

As simulações foram realizadas através de códigos implementados no MATLAB, no qual foi utilizada uma malha com 204 elementos em $x$ por 23 elementos em $y$, tendo a viga $1 m$ de comprimento por $0,1 \mathrm{~m}$ de altura. O material empregado para extrair as constantes constitutivas para a viga engastada foi o Alumínio, com módulo de Young $E$ igual a $65 \times 10^{9} \mathrm{GPa}$, densidade $\rho$ igual a $2890 \mathrm{~kg} / \mathrm{m}^{3}$ e coeficiente de Poisson $\nu$ igual a 0,334 [5]. Para as considerações a seguir, é importante lembrar que, neste trabalho, considerou-se a análise de vibrações livres e, portanto, tanto o vetor de forças $f$ quanto o amortecimento, dado pela matriz $C$, são nulos. Os gráficos apresentados nesta seção são referentes ao primeiro modo de vibração da viga. A viga sem buracos está representada na Figura 1. Já na Figura 2 são apresentados, respectivamente, a amplitude da vibração, com frequência de 76,15 Hz, obtida pela Transformada de Fourier, e o deslocamento, sendo este último obtido pela integração do sistema de equações dado em (14) e os deslocamentos na forma da Equação (8). Para fins de análise, considerou-se para todos os casos, o deslocamento na direção $y$ do último nó da discretização, sendo este o do canto inferior no final da parte livre da estrutura.

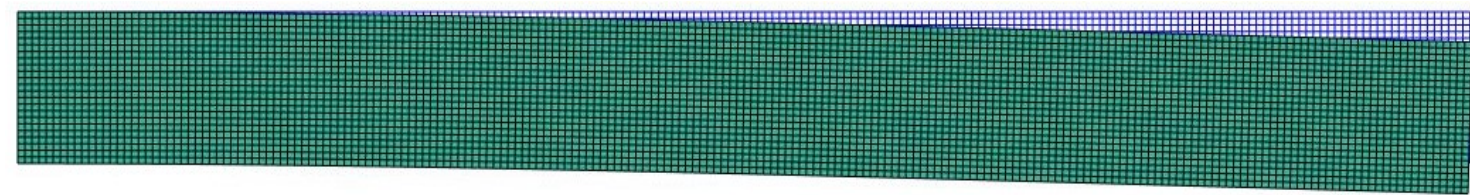

Figura 1: Viga sem buraco e seu primeiro modo de vibração. 


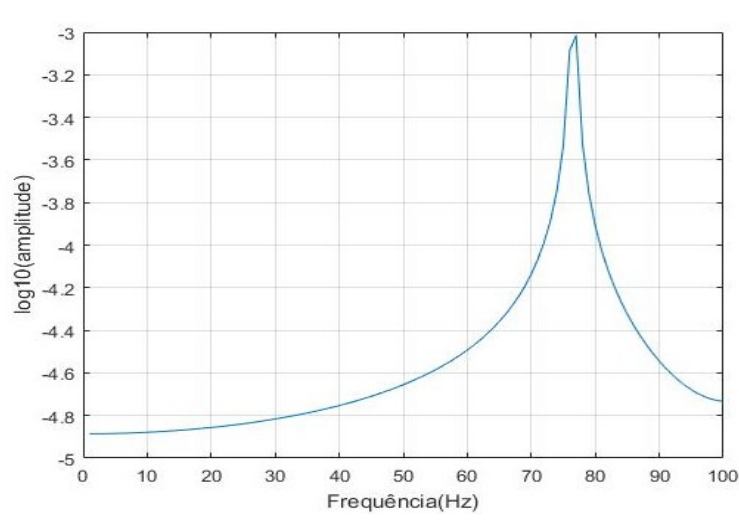

(a)

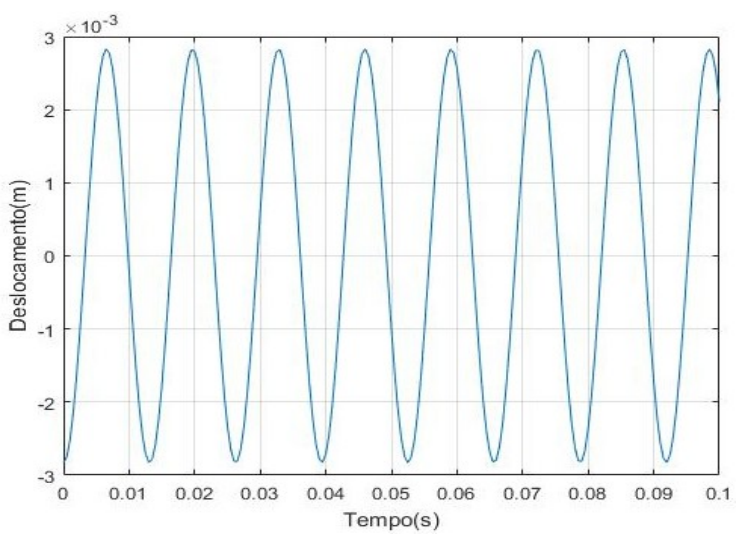

(b)

Figura 2: Resultados para a viga sem buracos prescritos. (a) Amplitude de vibração. (b) Deslocamento (vibração).

As condições iniciais para todos os casos em análise são $\xi=0,1$ e $\dot{\xi}=0,1$.

Na sequência, seguem os gráficos referentes às vigas com 1, 2 e 3 buracos prescritos, tendo como frequências $38,21 \mathrm{~Hz}, 44,66 \mathrm{~Hz}$ e $47,53 \mathrm{~Hz}$, respectivamente.
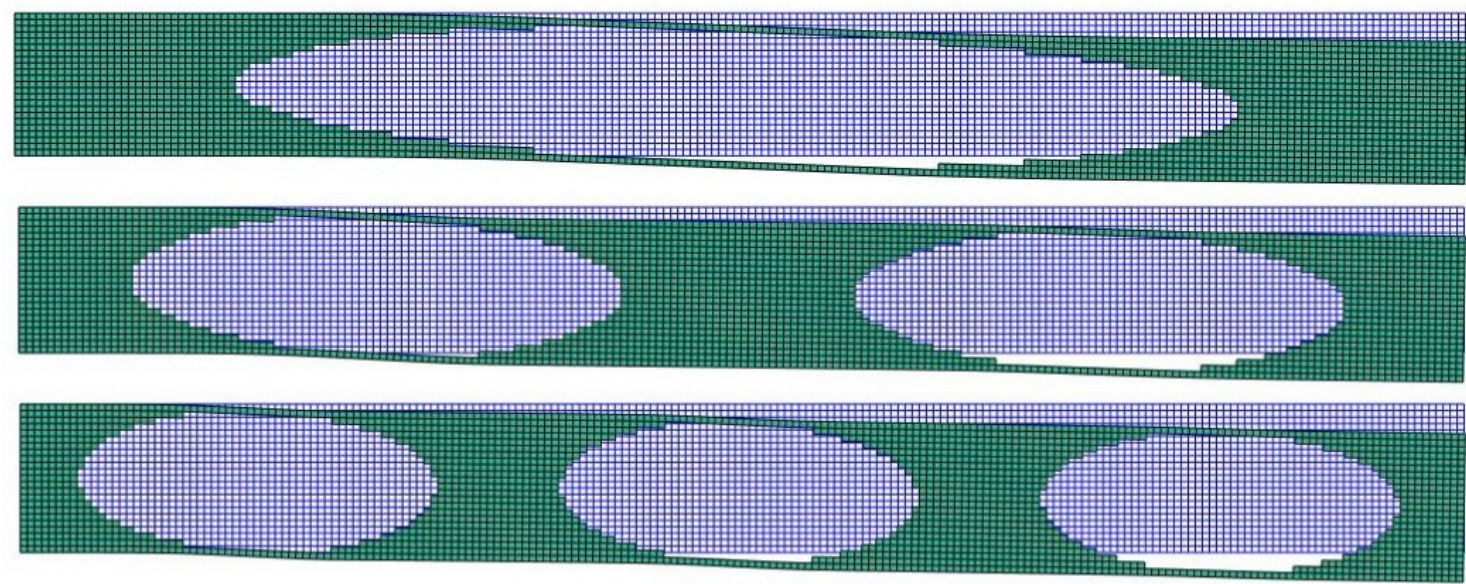

Figura 3: Vigas com 1, 2 e 3 buracos prescritos e seus respectivos primeiros modos de vibração.

Os gráficos da amplitude correspondente a cada frequência acima (todas calculadas pela Transformada de Fourier) estão apresentados na Figura 4, sendo a amplitude de vibração da viga com um buraco igual a 0,0006 m, com dois buracos igual a 0,0012 $\mathrm{m}$ e com três buracos igual a $0,0011 \mathrm{~m}$. 


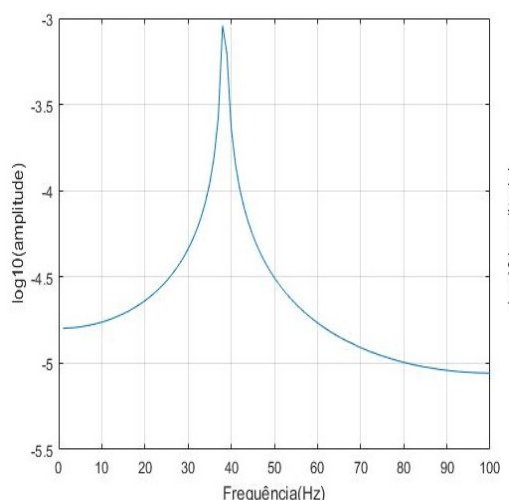

(a)

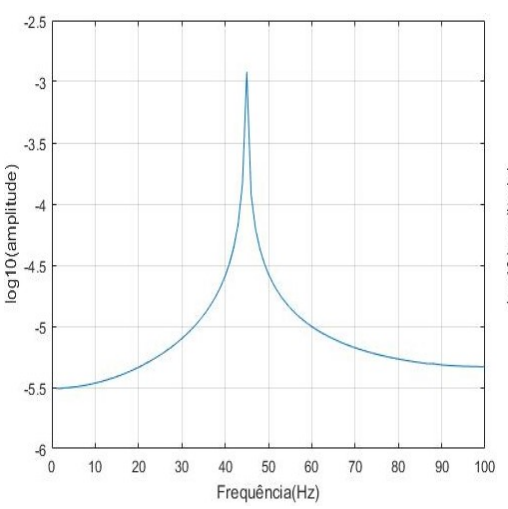

(b)

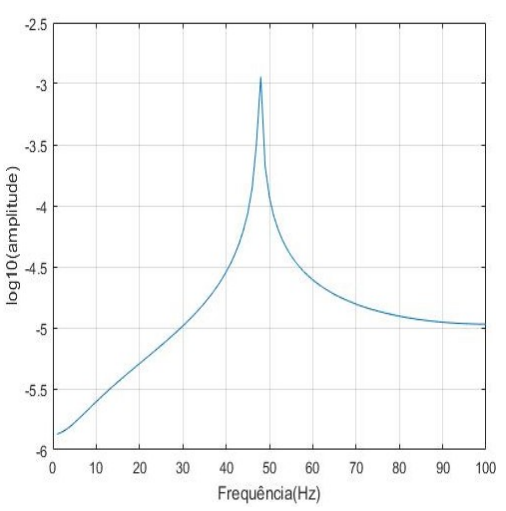

(c)

Figura 4: Amplitudes de vibração referentes às vigas com (a) 1 buraco; (b) 2 buracos; e (c) 3 buracos prescritos.

Pode-se observar pela Figura 4 que a configuração que apresenta somente um buraco foi mais satisfatória em comparação as demais configurações consideradas neste trabalho, uma vez que se obteve uma amplitude de vibração menor.

A Figura 5 apresenta os gráficos do deslocamento referente a cada uma das vigas apresentadas na Figura 3, no qual pode-se verificar, novamente, que a configuração com um único buraco prescrito é mais satisfatória em relação às demais configurações de buracos, por ter menor amplitude de vibração (embora a visualização desta diferença, nesta figura, seja menos perceptível do que na anterior).

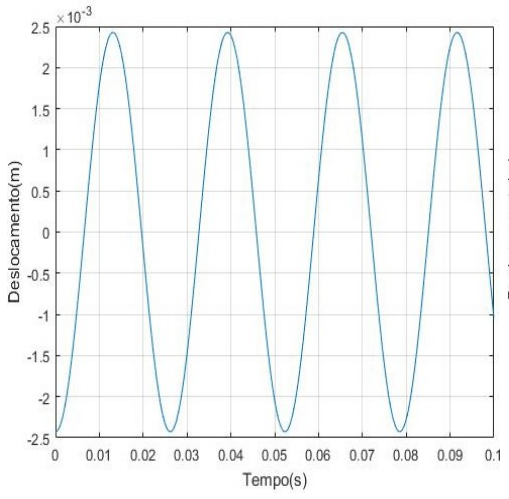

(a)

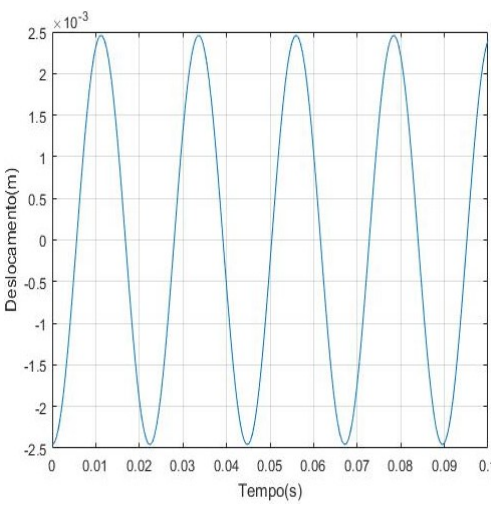

(b)

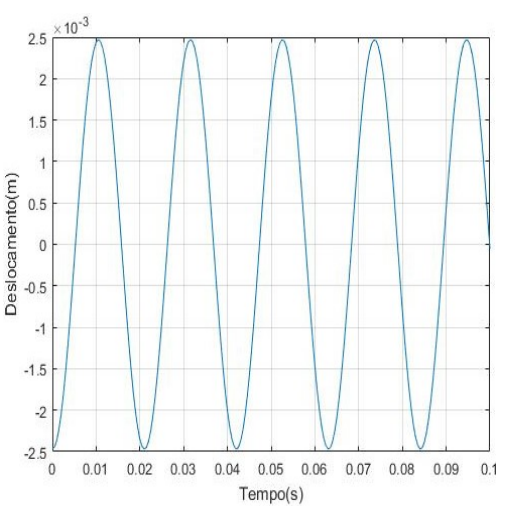

(c)

Figura 5: Deslocamentos (vibrações) referentes às vigas com (a) 1 buraco; (b) 2 buracos; e (c) 3 buracos prescritos. 


\section{Conclusões}

Neste trabalho foram analisadas as vibrações em uma viga em balanço com buracos prescritos. Consideraram-se vigas sem buracos bem como configurações apresentando 1, 2 e 3 buracos elípticos e centralizados. Utilizou-se o MEF para discretização do domínio da estrutura e cálculo dos deslocamentos nodais e a análise modal para a obtenção dos modos de vibração.

Levando-se em conta os resultados obtidos por simulações computacionais, foi possível constatar que a configuração que apresentou a menor amplitude de vibração foi a viga com somente um buraco.

Com este resultado, pretende-se, como sequência deste trabalho, fazer esta mesma análise para 2 e 3 modos de vibração, e utilizar esta configuração estrutural como controle passivo de vibrações e uma possível localização para a colocação de coletores de energia.

\section{Agradecimentos}

O primeiro autor agradece ao Conselho Nacional de Desenvolvimento Científico e Tecnológico $(\mathrm{CNPq})$ pelo apoio financeiro. A segunda autora agradece à Coordenação de Aperfeiçoamento de Pessoal de Nível Superior (CAPES) pelo apoio financeiro.

\section{Referências}

[1] Bendine, K., Hamdaoui, M. and Boukhoulda, B. F. Piezoelectric energy harvesting from a bridge subjected to time-dependent moving loads using finite elements. Arabian Journal for Science and Engineering, 44:5743-5763, 2019.

[2] Denis, V. Vibration damping in beams using the acoustic black hole effect, Tese de Doutorado, Université Du Maine, 2014.

[3] He, M., Zhang, X., Fernandez, L. S., Molter, A., Xia, L. and Shi T. Multi-material topology optimization of piezoelectric composite structures for energy harvesting. Composite Structures, $265(113783), 2021$.

[4] Liu, G.R. and Queek, S.S. The Finite Element Method: a practical course. Butterworth Heinemann, Oxford, 2003.

[5] Molter, A., da Silveira, O. A. A., Fonseca, J. S. O., Bottega, V. Simultaneous piezoelectric actuator and sensor placement optimization and control design of manipulators with flexible links using SDRE method. Mathematical Problems in Engineering, 2010(362437), 2010.

[6] O'Boy, D. J., Krylov, V. V. and Kralovic, V. Damping of flexural vibrations in rectangular plates using the acoustic black hole effect, Sound and Vibration, 329(22):4672-4688, 2010.

[7] Padoin, E. Otimização topológica de cascas compostas laminadas com atuador piezoelétrico para o controle de vibrações, Tese de Doutorado, UFRGS, 2014.

[8] Zhao, C. and Prasad, M. G. Acoustic black holes in structural design for vibration and noise control. Acoustics, 1(1):220-251, 2019. 